\title{
Commissioning the ATLAS Silicon Microstrip Tracker
}

\author{
Garcia, J. E. ${ }^{a}$ \\ on behalf of the ATLAS Collaboration \\ aUniversité de Genève, 24 Quai Ernest-Ansermet, 1211 Genève 4, Suisse \\ e-mail: jose.enrique.garcia@cern.ch
}

\begin{abstract}
The completed SemiConductor Tracker (SCT) has been installed inside ATLAS. Tests were performed last year to verify the connectivity of the electrical and optical services. Problems observed with the heaters for the evaporative cooling system have been resolved. This has enabled extended operation of the full detector under realistic conditions. Calibration data has been taken and analyzed to determine the noise performance of the system. In addition, extensive commissioning with cosmic ray events has started. The cosmic muon data has been used to align the detector, to check the timing of the front-end electronics as well as to check hit efficiency of modules. The current status of the SCT will be reviewed.
\end{abstract}

\section{INTRODUCTION}

ATLAS is a multi-purpose particle detector installed at the CERN Large Hadron Collider to exploit the physics of proton-proton collisions with $14 \mathrm{TeV}$ center of mass energy and luminosity of $10^{34} \mathrm{~cm}^{-2} \mathrm{~s}^{-1}$ [1]. The inner detector (ID) is a high performance tracker, prepared for the LHC very severe conditions. The main problems for the ID will be the large occupancies and high radiation doses. The design has been optimised to overcome these difficulties and give a precise tracking of particles. Some of the specifications and capabilities will be shown in this chapter. A most detailed description of the inner detector of ATLAS can be found in [2].

The inner detector is dedicated to track charged particles from the collisions. The ID combines high spatial resolution in the inner part: silicon pixels and micro-strip detectors; with continuous detection, transition radiation detection, in the external part. These subsystems are enclosed inside a solenoidal magnet with a 2 Tesla magnetic field, used for charged particles momentum measurement. Inner detector covers up to $|\eta| \leq 2.5$.

ID consists of three units: a barrel part extending over $\pm 80 \mathrm{~cm}$, and two identical end-caps covering the rest of the cylindrical cavity. Figure 1 shows a drawing of a $\phi$-section of one half of the inner detector. Starting from the most internal, the sub-systems that compose ID are: Pixels detector, Semiconductor Tracker (SCT) and Transition Radiation Tracker (TRT).

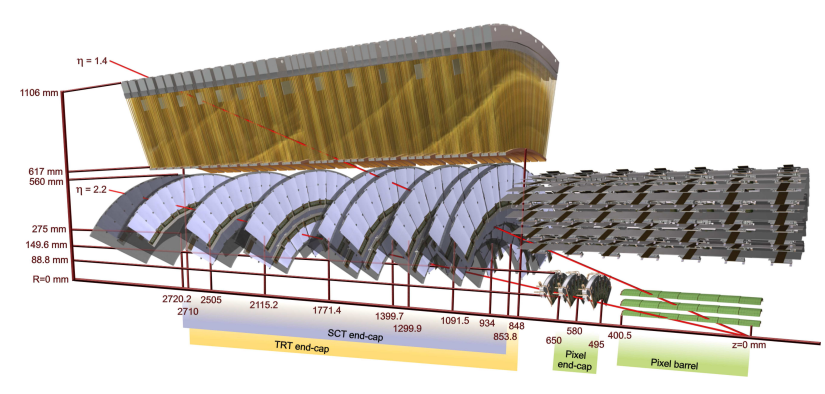

Figure 1. Drawing showing a view of the inner detector of ATLAS composed of: Pixel detector, Semiconductor Tracker (SCT) and Transition Radiation Tracker (TRT).

\section{SEMICONDUCTOR TRACKER}

The Semiconductor Tracker (SCT) provides four precision space points in the intermediate radii of the inner detector. Silicon micro-strip detectors cover large area $\left(61.1 \mathrm{~m}^{2}\right)$ with 6.2 million 


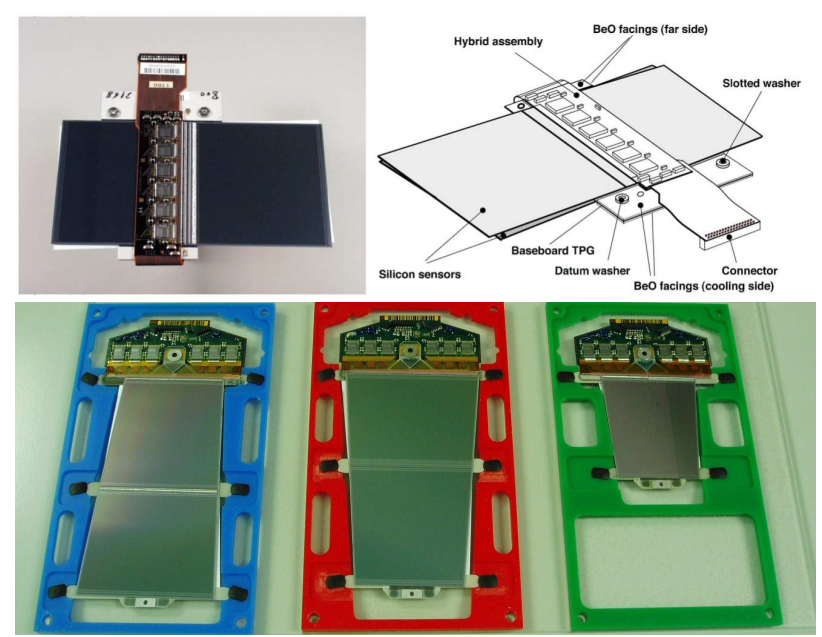

Figure 2. On top a photograph (left) and drawing (right) of a barrel module, showing its components. The thermal pyrolytic graphite (TPG) base-board provides a high thermal conductivity path between the coolant and the sensors. Bottom, photograph of the three end-cap module geometries: outer, middle and inner.

of readout channels.

Four cylindrical barrels (2112 modules) with a pseudo-rapidity range going up to $|\eta|=1.1$ and $|\eta|=1.4$ depending on the barrel, whereas two end-caps (1976 modules) with nine wheels each extend the acceptance to $|\eta|<2.5$. The inner radius of the $S C T$ is $30 \mathrm{~cm}$ from the interaction point, the outermost layer is at $56 \mathrm{~cm}$. The barrel goes to a distance along the beam of $80 \mathrm{~cm}$ in both directions. Nine wheels with different inner radii provide measurements in the region from $|z|=81$ to $|z|=279.7 \mathrm{~cm}$.

Each SCT module (Figure 2) consists of two planes of silicon micro-strip detectors glued back to back with a $40 \mathrm{mrad}$ angle between them [3] [4]. This module design allows a space-position measurement with precision of $17 \mu \mathrm{m}$ in $R \phi$ and $580 \mu \mathrm{m}$ in $Z$ plane. Each module is read out by 12 ABCD3T ASIC chips, constructed in radiation hard technology DMILL. The chips are mounted on $\mathrm{Cu}$ /polyimide hybrid flex circuits and incorpo- rated on the module itself [5]. Each ABCD chip provides sparsified binary readout of 128 detector channels. The hit information from each channel is stored in a 132-deep binary pipeline buffer. The chips also contain a charge injection circuitry used for calibration. The readout has implemented redundancy at different levels (chips and modules) [5].

The modules are required to survive an integrate fluence of $2 \times 10^{14} 1 \mathrm{MeV}$ neutronequivalent $/ \mathrm{cm}^{2}$, corresponding to 10 years of LHC operation. To reduce the leakage current, to prevent reverse annealing and maintain and adequate noise levels after radiation damage the sensors will be kept at $-7^{\circ} C$. Without proper cooling, the damage in the detectors increases the depletion voltage further due to reverse annealing. The power consumption is $5.5 \mathrm{~W}$ per module initially, which will increase on around $1 \mathrm{~W}$ per module due to leakage current after 10 years of operation. The heat generated is extracted by an evaporative cooling system employing $C_{3} F_{8}$. The temperature of the coolant in the cooling pipes has to be kept at about $-25^{\circ} \mathrm{C}$ to achieve the desired temperature in the modules.

\section{SCT COMMISSIONING}

The macro-assembly of the SCT was performed in four assembly sites and finished in 2005. The four barrel cylinders were assembled in Oxford University and CERN. The two end-caps were assembled in Liverpool and NIKHEF. The modules and services were mounted on the carbon-fibre support structures. The services include the optical communication system, the electrical lines using Low Mass Tapes (LMTs), and the Frequency Scanning Interferometry (FSI) alignment system.

The barrel was inserted in the cavern in August 2006. The barrel went through optical, electrical and cooling tests. The barrel was signed-off in April 2007, and in May and June 2007 both end-caps were inserted into ATLAS cavern. The end-cap sign-off happened during February 2008. During the sign-off period and after, several tests were performed. An important check is the measurement of the noise occupancy. Figure 3 shows the distribution of noise occupancy per channel 
and bunch crossing, averaged over a module side (768 channels) measured for all active module sides of the barrel and end-cap cosmic runs. Endcap inner modules are not shown on the plot as their noise occupancy is typically $<10^{-7}$. Results are in very good agreement with values obtained at the assembly sites.

After the sign-off of the whole system the SCT joined for the first time the ATLAS combined Milestone run (M6) in March 2008. The detector was successfully integrated with the central DAQ and took part in the following cosmic data taking.

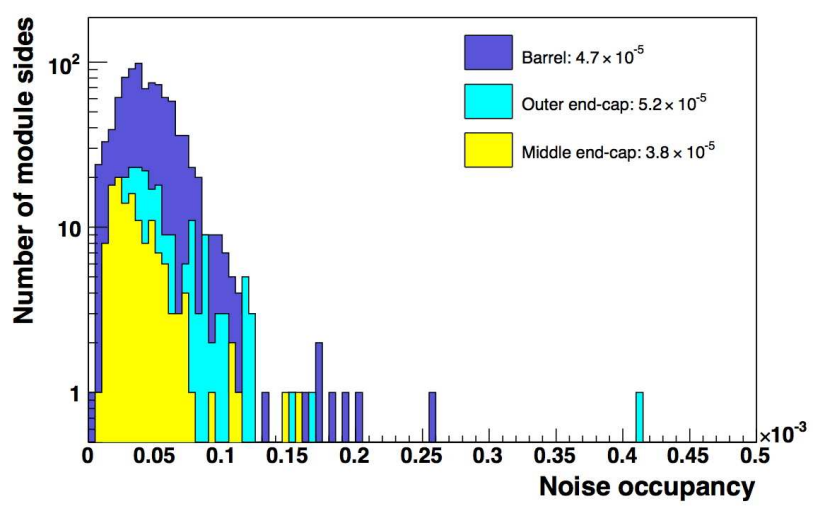

Figure 3. Distribution of the average noise occupancy for barrel, outer and middle modules

In May 2008 failure in the cooling plant which affected 3 of the compressor stopped the SCT operation up to the end of August when the plant was back on. Optical tuning and calibration were performed immediately after the cooling was recovered. The calibration effort made possible that the system was ready for the LHC turn-on on 10th of September. All but two cooling loops, one in Disk 9, and one in Disk 1 are running. The two not cooled loops service 36 of 4088 modules. ${ }^{1}$.

${ }^{1}$ One of those two cooling loops servicing 23 modules will be repaired during the shutdown.
To operate with the ATLAS system, the SCT had to be synchronized to the trigger signals and to the other sub-systems. In order to achieve this a set of scans with different global delay offset was performed in cosmic data taking. A good synchronization results in an increase on the number of space-points and tracks. The optimal offset, once it was found, was kept for the rest of the cosmic running. A finest synchronization is still needed, but this requires beam data, thus it will have to wait up to next beam run in 2009 .

After the brief run with beams in LHC data taking continued in ATLAS with cosmic muon runs. An extensive period of cosmic data taking with and without magnetic field started. During approximately two months, more than one million of cosmic tracks have been collected in the SCT detector. This period has been extremely useful to develop and debug the data acquisition (DAQ), Detector Control System (DCS), monitoring systems and verify the offline data processing. At the beginning of October for the first time the pixel system was included in the data acquisition together with the SCT and TRT. Figure 4 show an example of a track collected and seen by the pixels and SCT with magnetic field off.

The large number of cosmic tracks collected allows the alignment of the barrel section. Due to the geometry of the cosmic trigger and the nature of the cosmic rays themselves, only the barrel system can be aligned with a good precision. Results show similar residuals as obtained in the March 2008 cosmic test, with only a $<50 \mu m$ shift between the solenoid field being on and off. Work is ongoing to improve the alignment with the total collected data.

One of the problems that has been faced during November 2008 is the high rate of deaths of the VCSELs used to transmit commands and clock to the modules. The same VCSEL technology has been used in the modules and no failure rate has been observed in there. The cause of the failure is suspected to be Electro Static Discharge (ESD) damage during the handling of the optical hardware. Fortunately these VCSELs are located in crates in the service caverns so they can be replaced. Just recently (middle of November) most of the damaged VCSELs have been replaced. The 
default configuration of the SCT includes:

- Barrel: $99.1 \%$

- End-cap A: $98.7 \%$

- End-cap C: 36 of 988 modules are not cooled. Percentage of modules included in default readout without considering not cooled modules is $98.9 \%$ being $95.3 \%$ if they are taken into account.

Several of those modules are not readout because of off-detector system problems which will be fixed during this shutdown.

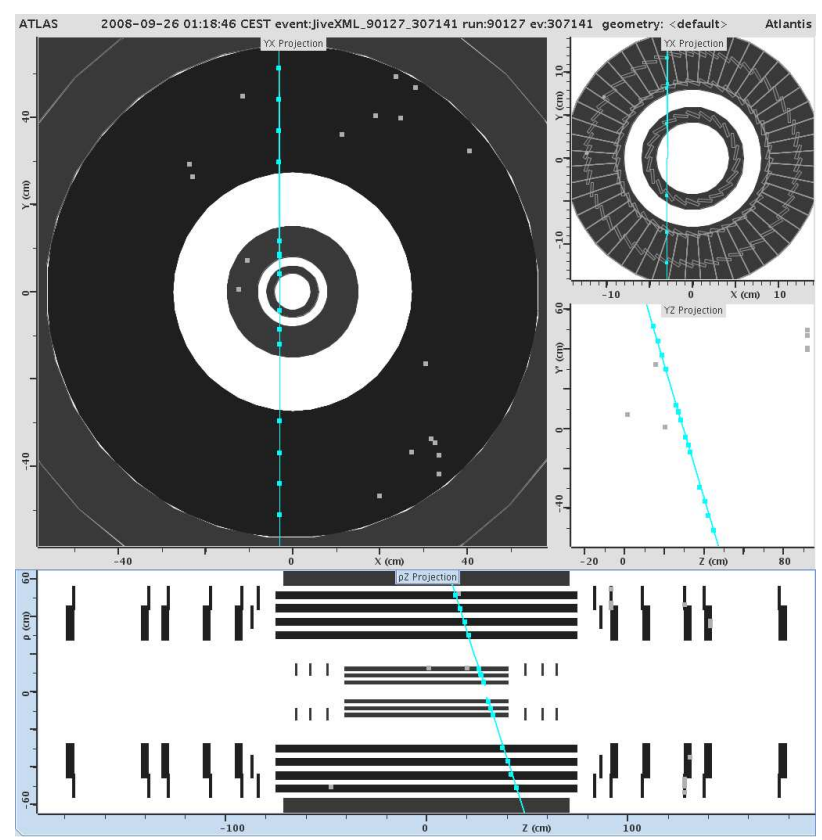

Figure 4. Reconstructed cosmic track seen by the pixels and SCT systems. Magnetic field was off for this run.

In parallel during this cosmic period a round of calibration and tuning of modules took place. A deeper calibration has been done to improve the performance of every single module (reduce noise, improve light output,...). The system has been optimized and it is now ready for the last combined cosmic run that will take place at the end of November (starting November 26th). This combined run will be a good opportunity to test all the improvements in calibration and repairs.

\section{CONCLUSION}

The SCT was installed in the ATLAS cavern, tested and officially signed-off early in 2008. The SCT has successfully been integrated with the ATLAS DAQ system and joined the combined Milestone runs during 2008. Once the cooling plant was recovered in August 2008, the SCT started operation again.

SCT was ready to take data during the first beam in September. For that first beam, for safety reasons sensors and electronics were operated at reduced voltage (barrel was off and endcap at $20 \mathrm{~V}$ sensors). Despite the reduced voltage, signal was observed in the end-caps for the splash events (beam colliding into a collimator producing a shower of muons) and beam halo. Unfortunately collisions will not happen up to next year once the LHC has been repaired after the accident on September 19th. The SCT has gone through a thorough calibration and the system is now ready and in an excellent shape, looking forward for the first collisions next year.

\section{REFERENCES}

1. The ATLAS Detector and Physics Performance Technical Design Report, CERN/LHCC 99-14, 1999.

2. The ATLAS Collaboration, G. Aad et al., The ATLAS Experiment at the CERN Large Hadron Collider, JINST 3 (2008) S08003

3. A. Abdesselam et al., Nucl. Instrum. and Methods Phys. Res. A 568 (2006) 642-671

4. A. Abdesselam et al., Nucl. Instrum. and Methods Phys. Res. A 575 (2007) 353-389

5. F. Campabadal, et al., Nucl. Instr. and Meth. A $552(2005) 292$. 